\title{
Design, Simulation and Fabrication of a New Finger Support
}

\author{
Simin Nasseri" ${ }^{\#}$, Vitale Kyle Castellano\#, Tammy Ong\#, Anthony DeDiego ${ }^{\#}$ and Nasrin Naseri^ \\ \#Kennesaw State University, Southern Polytechnic College of Engineering and Engineering Technology, Mechanical Engineering Department, USA \\ ${ }^{\wedge}$ Tehran University of Medical Sciences and Health Services, School of Rehabilitation, Department of Physiotherapy, Iran
}

Received 06 May 2018, Accepted 09 July 2018, Available online 10 July 2018, Vol.8, No.4 (July/Aug 2018)

\begin{abstract}
This paper reports on the results of design and fabrication of a new finger support. Finger deformation is very common and has many causes and severity. Most of the available finger supports are not resizable and comfortable. They are mainly designed to fully restrict the finger's motion when there has been any type of injury. However, for example in the case of early stages of arthritis, the patient should be able to use them whenever they want. Furthermore, the available finger supports cannot be adjusted considering various fingers' sizes and positions. In this research, seven models of a polymer-metal composite finger support are designed. They are made of a soft polymer with inserted sheets of aluminum, steel or carbon-fiber. The optimal models are strong, and allow for size and finger position adjustments, and can be used for patients who already have distorted fingers and are working on them to regain some functions. Extensive finite element analysis of the support under the distributed loads of the finger, confirmed by the results obtained by a MATLAB program, shows that the new support tolerates the applied forces without any permanent deformation. Finally, the fabricated part using 3D printing validates the results.
\end{abstract}

Keywords: Arthritis; Polymers; Composite; 3D printing; Finite Element Analysis; Modeling.

\section{Introduction}

Finger deformity or abnormality can occur in numerous ways which are related to many diseases such as Rheumatoid Arthritis (RA) and Osteoarthritis (OA) (Egger,1995), or body conditions which are hereditary and so on. Some of these are flexion deformity (Vasiliadis, 2017), (Wang, 2017), mallet finger (Valdes, et al, 2015), Dupuytren's disease (Foucher, 2001), boutonniere deformity (Palchik, 1990), (Williams, 2011), swan neck deformity (Giesen, 2009), valgus deformity (Deveci, 2015), and clawfinger (Brandsma, 2015), etc.

Finger deformity related to many of these conditions causes a noticeable curved or bent finger that is unable to straighten on its own without splinting, physical therapy and other surgical and nonsurgical methods. Each of these conditions have a different root cause and its severity varies based on the condition. One form of treatment is to wear a finger support that will allow the finger to take the shape of its support gradually. This type of treatment will slow the progression of deformity without surgery or other costly treatments, and in some cases, correct the finger

*Corresponding Author's Mail snasser1@kennesaw.edu (ORCID ID:0000-0002-6036-053X)

DOI: https://doi.org/10.14741/ijcet/v.8.4.9 deformity and improve its function. Table 1 describes different deformities, the cause of the deformities and their effect on fingers.

It is important to mention that the supports are considered preventive, corrective, or over-corrective. For instance, for patients suffering from inflammatory joint diseases, a support which would accommodate the resting position is recommended. In many cases a corrective or over-corrective support is advised especially when the patient's finger is severely deformed. In such cases, placing the finger in a functional position is suggested so that the patients would be able to do their normal life tasks. The functional position of the hand fingers consists of dorsiflexing both the wrist between $20^{\circ}-35^{\circ}$ and the Proximal Inter Phalangeal (PIP) joints between $45^{\circ}$. $60^{\circ}$. The thumb is abducted and in opposition and alignment with the pads of the fingers (Wang, 2017). In some cases, an over-corrective position for the finger is advised, in which the support is used to straighten the finger and correct its abnormal curvatures.

There are some finger supports that exist, but these supports are bulky, made of uncomfortable materials and are not able to accommodate to different conditions and deformities. Some even cause irritation when having to wear them for long periods of time. They do not allow users to partake in everyday activities and can make basic tasks challenging. 
Table 1 Finger deformities, causes, description, and effects on finger

\begin{tabular}{|c|c|c|}
\hline \multicolumn{3}{|c|}{ Finger Deformities, Causes and Effects } \\
\hline Name & Cause/ Description & Effect on Fingers \\
\hline Flexion deformity & $\begin{array}{l}\text { The flexor tendon becomes inflamed and } \\
\text { cannot move smoothly inside the sheath } \\
\text { (trigger finger). }\end{array}$ & $\begin{array}{l}\text { Metacarpal phalangeal (MCP), PIP, distal } \\
\text { interphalangeal (DIP) are bent sequentially. }\end{array}$ \\
\hline Mallet Finger & $\begin{array}{l}\text { Loss of the extensor mechanism at the DIP } \\
\text { joint. Also known as baseball finger or drop } \\
\text { finger. }\end{array}$ & $\begin{array}{l}\text { DIP bends specially in the ring finger } \\
\text { (baseball finger). }\end{array}$ \\
\hline $\begin{array}{c}\text { Dupuytren's contracture or } \\
\text { disease }\end{array}$ & $\begin{array}{l}\text { Hereditary disorder affecting mainly male } \\
\text { patients. }\end{array}$ & $\begin{array}{l}\text { The fingers will bend closer to the palm on } \\
\text { the hand. }\end{array}$ \\
\hline Boutonniere Deformity & $\begin{array}{l}\text { Due to joint inflammatory diseases (RA or } \\
\text { OA) or extensor mechanism rupture. }\end{array}$ & $\begin{array}{l}\text { It causes the PIP joint to bend inward } \\
\text { towards the palm (flexion) and the DIP joint } \\
\text { to bend outward (extension). }\end{array}$ \\
\hline Swan Neck Deformity & As above & $\begin{array}{l}\text { PIP joint bends outward and the DIP joint } \\
\text { bends inward towards the palm. }\end{array}$ \\
\hline Valgus deformity & $\begin{array}{l}\text { RA or OA. Also, Hallux Valgus caused by using } \\
\text { narrow-toe shoes. Besides, abnormal } \\
\text { congenital joint surfaces of the big toe. }\end{array}$ & $\begin{array}{l}\text { The big toe will deform and move in the } \\
\text { direction of the other toes. }\end{array}$ \\
\hline Clawfinger & $\begin{array}{l}\text { Nerve damage caused by diabetes or } \\
\text { alcoholism, etc. leading to drop wrist or drop } \\
\text { foot. }\end{array}$ & $\begin{array}{l}\text { The MCP joint bends outward and the other } \\
\text { joints bend inward. }\end{array}$ \\
\hline
\end{tabular}

Different studies accessed supports made of different materials which cover the finger in different ways (Figure 1A). Some supports are made of a thermoplastic that covers the circumference of the finger and some are padded piece of aluminum that has different ways of fastening the device (Valdes, et al, 2015).

Valdes, et. al. compared different types of support/splint devices for patients with mallet finger (Figure 1A). Padiachee and his coworkers, used dental putty, hard plaster, carver, and cold cure acrylic (Figure 1B) to create the supports (Padiachee, et al, 2016). The support was claimed to be inexpensive, and lightweight, yet it is not resizable and does not accommodate various finger positions (resting, and functional).
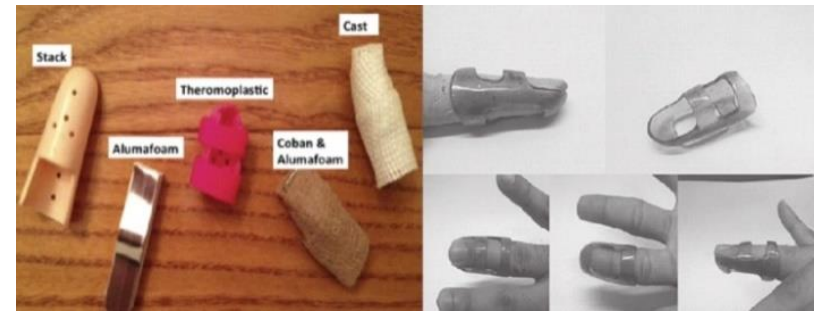

Fig. 1 On the left (1A): Different supports for patients with mallet finger (1B) (Valdes, et al, 2015). Different views of the device made of dental impression putty

(Padiachee, et al, 2016)

In research work of Colbourn and her coworkers, a finger splint was made out of a thermoplastic polymer to treat severe trigger finger (Colbourn, et al, 2008). The design created is simple and easy to fabricate, yet it lacks the same capabilities mentioned before.

The main goal of this study is to design and fabricate a new device which is not only comfortable, but also light and durable. It is also important for the finger support to be able to accommodate many different shapes and sizes of fingers. Additionally, it is also beneficial that the support provides various finger positions, for example providing a functional position for fingers is necessary and is not addressed elsewhere.

There are three major types of polymers which include: thermoplastics, thermosets, and elastomers (Groover, 2010). Unlike thermosets, when thermoplastic polymers are subjected to heating and cooling cycles, there is not significant degradation. Elastomers (natural rubber, synthetic rubber, and thermoplastic elastomers) have excellent elasticity and extensibility. For the purpose of this paper, an elastomer will create the best material to use because of its elasticity and ability to be flexible. Elastomers are mainly soft and will not cause irritation on the skin. In 3D printing, soft polymers commonly used are

TangoPlus, TangoBlackPlus, Agilus30, and Agilus30Black. These materials, have rubber-like qualities, flexible, and are soft to the touch.

In this research, seven composite models were designed and evaluated, in which two models were fabricated. One of these models allow the patients to use the support in the functional position, letting them do their normal life tasks. The designs differ in strength, and adjustability. The finger support can also vary from the composition of the soft polymer with different types of metal, such as aluminum, steel or carbon fiber.

The fabrication aspect of the new finger support can range from extrusion to using $3 \mathrm{D}$ printing. Compression molding is a common method for manufacturing rubber and elastomer material and is a less costly process compared to injection molding (Groover, 2010). In this method of manufacturing, a mold is created and then the polymer is pressed into its form (Han, 2014). By using 3D printing, one can fabricate the device quicker and cheaper than through compression molding or extrusion. In case of bulk manufacturing, other methods can be utilized. 
In this paper, the results of modeling (including the calculated maximum stress and maximum deformation) for one of the models are presented. The maximum stress values were compared with the yield strength values of the metals used to ensure that the deformations were not plastic or permanent. Furthermore, to validate the modeling results, two supports were fabricated and tested which confirmed that the new finger support in this work is reliable, useful, and durable.

\section{Materials and Methods}

Finding the finger's force: The motion of the human finger is important to determine the finger function and to calculate the maximum force the finger creates. The maximum force of a finger will determine the amount of stress and strain that will be put on the polymer and metal (Arslan et al 2008) determined the trajectory of the finger model, which mimicked the motion characteristics of the human hand during closing and opening motion. They developed a biomechanical model of the index finger of the human hand, based on the human anatomy, to create a prosthetic hand. They created a system of partial differential equations to depict the forces of a finger to move to a certain position.

Furthermore, some researchers measured the flexing force of fingers using a dynamic sprint with a dynamometer (Silva, et al, 2005). The purpose of research was to determine the forces that act on the joints. A dynamic splint was created and was linked to a dynamometer that measured flexing force. To calculate the flexing force trigonometric equations were used and data was collected with varying angles ranging from $25^{\circ}-45^{\circ}$.

Hofmann and Goebl investigated the finger force profiles and tongue techniques of clarinet students and professional clarinetists under controlled performance conditions. According to their methodological study conducted on finger force when playing some instruments, a healthy finger force varied with different test subjects of different body types and sex. Gathered from the research studies (Hofmann and Goebl, 2016), an average finger flexion force can be considered as $120 \mathrm{gf}$ which converts to $0.2644 \mathrm{lbf}$ or $1.177 \mathrm{~N}$. However, they also expressed that the largest finger force during the high expression level performance conditions was increased to $1.21 \mathrm{~N}$ which is considered for this present work. This is consistent with other researcher's findings who used dynamometers (Silva, et al, 2005), (Wang, 2017).

Various Models: Overall, seven models have been designed in this study. These supports could be worn at resting or functional positions mentioned before. Some of the models can be modified for functional position and some are already designed for that purpose. Each model is designed to improve the previous model or add some new functionality.
In model one, fiber reinforced polymer (FRP) is used, whereas on others, the composite models are designed by using a polymer and strips of aluminum, steel, and carbon fiber. Model one (Figure 2A) is one of the simplest designs to create, as it is a simple cylinder, but incorporating metal fibers within the polymer makes it more difficult to manufacture. To fabricate this model, a multi-material 3D printer should be used to incorporate metal fibers. However, it can also be extruded. Model two (Figure 2B) is comprised of a cylinder with two vertical slots on opposite sides that will hold strips of aluminum metal. This model will be stronger and can take on the force of the finger when bending. Model three (Figure 2C) has a bigger diameter on the bottom and smaller diameter on the top to go with the angle of a finger. Considering the extrusion process, this design is more difficult to create, but can be made by applying heat treatment methods to model two.

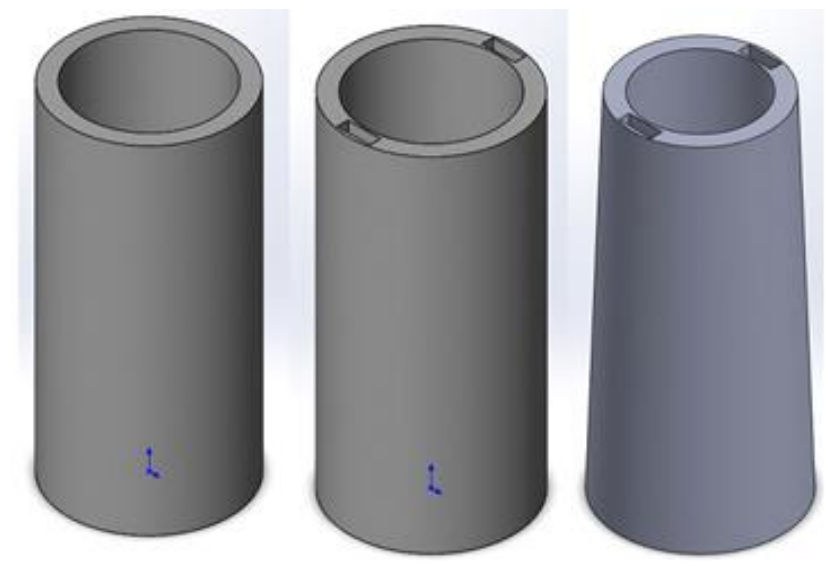

Fig. 2 On the left (2A)- Model 1 (polymer with metal fibers): An FRP model. In the middle (2B)- Model 2 (Slotted): Composite model of the elastomer and sheets of aluminum, steel or carbon fiber. On the right (2C)Model 3 (Tapered): Similar to model 2, but tapered

The design of model four (Figure 3A) accommodates the opening and closing of the support, hence the user will be able to adjust the finger support to fit the size of their fingers or make only the tip of the support narrower. It has two slots, one vertical and one horizontal for inserting strips of metal. Since each slot's dimensions allow insertion of only one strip of metal, two vertical strips are considered to be inserted from top and bottom (into the vertical slot) after the horizontal metal strip is inserted (Figure 3B). However this negatively affects the resistance of the vertical sheet against the applied force of the finger. To resist the finger flexion force, a complete sheet of metal should be inserted vertically. Furthermore, this model has thin areas which might be difficult to fabricate using 3D printing and is subjected to tear after being used for a long time. 


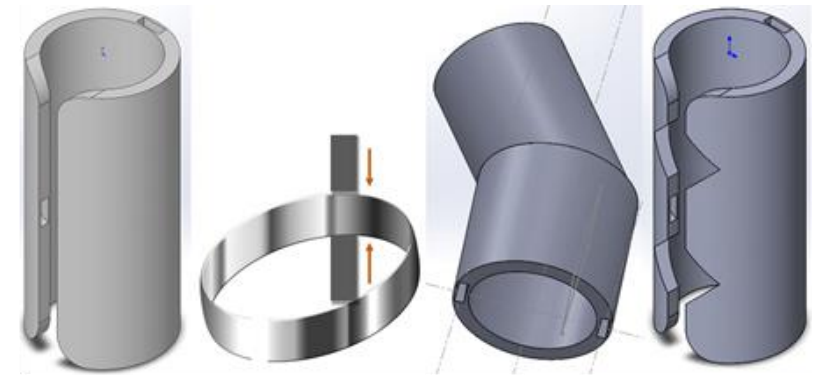

Fig. 3 On the left or 3A- Model 4 (Flexible): A cylinder with slots for three metal strips inserted vertically and horizontally (3B). Figure 3C- Model 5 (Angled): Suitable for patients who already have a severely bent finger. On the right or 3D: Model 6 (Versatile): A fully flexible model allowing the support to be adjusted for various bend angles

Model five (Figure 3C) is ideal for patients that are unable to fully extend their fingers in functional positions. Depending on the severity of the finger's deformity, this model can be redesigned at various angles to fit a finger at its maximum possible angle of extension. If a patient is attempting to strengthen the finger to eventually be fully extended again, the patient can "train" the finger by changing the support as often as directed to increase their finger's angle of extension each time the support is changed. Aluminum, steel, or carbon-fiber strips can still be inserted on either side of the model; however, this model requires two angled strips to give the support the required strength.

Model six (Figure 3D) is similar to model five but has a few adjustments, such as having slits in the back of the support and triangle shaped cuts in the front where the support opens and closes. The purpose of the slits is for it to be bendable and to adjust to more positions. This support is slightly lighter compared to model four. Alternatively, for ease of manufacturing and reducing the cost, it can be manufactured as it is flatted out and then become cylindrical with the aid of the horizontal inserted metal. The issue with the second method is the springback of the polymer which is a typical phenomenon with viscoelastic materials. Because of the elasticity of the polymer, it tries to return to its original flat shape. So all of the four top and bottom edges will be distorted outward. This problem can be resolved by heat treatment and recrystallization (Groover, 2010).

Model seven is based off model four and addresses 3D printing limitations, which can be viewed in Figure 4. This model serves as one of the best in terms of strength, durability, comfort and ease of manufacturing when compared to others. Primarily it allows the circular hollow regions to be created during the 3D printing process which will use a resin material to fill these cavities during printing. The challenge of model four was the removal of this resin without causing damage to the concept. Model seven has a radial cut which allows the resin to be removed, yet it still retains the aluminum rings. This design also incorporates three rings for better support. These rings are also where the forces will be placed in finite element analysis (FEA). It should also be noted that the vertical sheet rests above the rings, but remain in contact with them. The vertical sheet has also been modified to be curved for better contact between it and the TangoPlus finger support. Lastly, a minimum wall thickness was assumed, considering the limitations of 3D printing This model can be redesigned to be suitable for functional position of the finger easily. Model seven was used in FEA and the results will be discussed below.
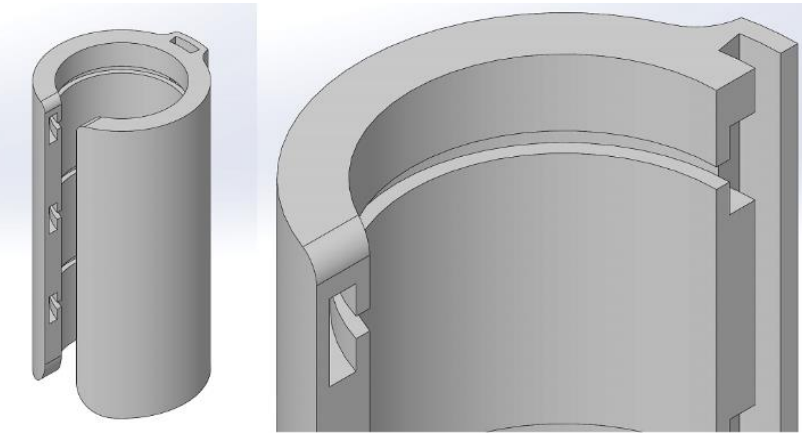

Fig. 4 On the left or 4A- Model 7 (Optimal): A composite model made of TangoPlus polymer, one vertical metal sheet and three metallic rings. On the right or 4B- Model 7 close up.

Governing Equations: The strip of aluminum that will be inserted in the polymer finger support can be treated as a cantilever thin plate, because there is no force or movement at the base of the support. As mentioned before (Hofmann and Goebl, 2016), the amount of maximum force exerted by fingers was found to be $1.21 \mathrm{~N}$, which is considered here.

Since the aluminum strip will be treated as a thin plate, the common formulas for bending stress and deformation can be applied. This is the base force which is considered for simulation and later it is shown that the support can tolerate forces much higher depending on the inserted metal in the support.

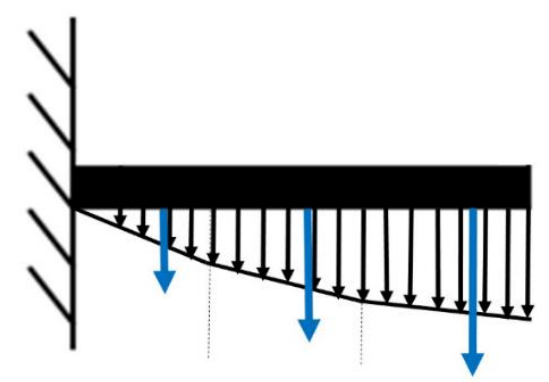

Fig. 5 Three distributed forces and their equivalent point forces for the finger

A finger exerting flexion forces, can be treated as a cantilever beam with either three distributed loads or with three-point forces concentrated proportionallyspaced along the length of the finger. Figure 5 above 
shows an image of a cantilever beam with three-point forces representing the three distributed forces. The forces are $1.21 \mathrm{~N}, 0.806 \mathrm{~N}$ and $0.403 \mathrm{~N}$, uniformly distributed along the length of the support.

For a finger exerting a force treated as a concentrated or point load at one point, the following equation can be used to find the deflection of the metal strip:

$\delta=\frac{F L^{3}}{3 E I}$

where $\mathrm{F}$ is the force in $\mathrm{N}, \mathrm{L}$ is the length of the strip in $\mathrm{mm}$ or distance from the base of the finger to the location of the equivalent point force being exerted, $\mathrm{E}$ is the elastic modulus of the metal (a measure of stiffness) in MPa and I is the mass moment of inertia or resistance against bending in $\mathrm{mm}^{4}$. For each of the three distributed loads shown in Figure 5, an equivalent point load is calculated using common tables of Engineering Mechanics textbooks. Also, aluminum 6061-T6 which has been selected for this research has a modulus of elasticity (E) of $69000 \mathrm{MPa}$. The moment of inertia (I) for the shape of this aluminum strip can be calculated with the rectangular cross section by $\left(b^{3}\right) / 12$, where $b$ is the width of the strip, and $t$ is the thickness both in $\mathrm{mm}$. One should notice that if the force of the finger were to be treated as a uniformly distributed load across the entire length of the metal strip, the following equation could be applied:

$$
\delta=\frac{q L^{4}}{8 E I}
$$

with $\mathrm{q}$ being the weight per unit length of the beam in $\mathrm{N}-\mathrm{mm}-1$. With these equations, one can calculate the amount of force the finger creates when bending treated as either a point force or distributed load. The amount of deflection the metal and polymer will experience when under a force can then be determined.

Here the conjugate beam method is used which is an engineering method to derive the displacement and slope of a beam. The load on the conjugate beam is the M/EI diagram of the loads on the actual beam. Hence, the following equations were used to validate the FEA process and results:

$\sigma=\frac{6 M}{b t^{2}}$

$\Delta=\frac{F a^{2}}{2 E I}\left(L-\frac{a}{3}\right)$

$\mathrm{M}$ is the moment produced by each force in $\mathrm{N}-\mathrm{mm}$, and $a$ is the location of the force on the beam relative to the fixed end in $\mathrm{mm}$.

A MATLAB (Mathworks ${ }^{\odot}$ ) program was written to predict the maximum deflection and maximum stress of a beam under three-point loads. The program is able to obtain all the input values from the user (parameters mentioned above) and give the output values. This program was helpful in terms of predicting the deflection and stress values for a metal beam and therefore validating the results which were obtained by simulation. The code also reported the factor of safety by considering a yield, or tensile, strength of the material versus the calculated stress to estimate how much the forces can be increased (Kurowski, 2016). Simulation: Model seven was considered for FEA using

SolidWorks ${ }^{\odot}$. The goal was to evaluate the performance of the entire model including the metal sheet (e.g. aluminum 6061-T6), three metal rings, and the TangoPlus support while under the applied load of the finger.

Through careful examinations and calculations, the thin areas in the support were detected and were modified, in simulation analysis, considering the limitations in 3D printing. To evaluate the effectiveness of the simulation, each von-Mises stress value had to converge to a value below the yield stress of each material used and the total deformation of the support ought to be within an appropriate range that the MATLAB program predicted.

Three-point loads were applied at the middle of the three rings from inside, which are in direct contact with the metal sheet. A fixture was applied only at one of the ends of the sheet to simulate a cantilever beam. The vertical sheet dimensions were $4.78 \mathrm{~mm}$ by $1.60 \mathrm{~mm}$ by $50.80 \mathrm{~mm}$. The three-point loads were 1.21 , 0.806 , and $0.403-$ Newtons located at 46.037, 30.163 and $14.287 \mathrm{~mm}$ from the fixed end of the sheet, respectively. These were calculated based on similar triangular rules. These values were given based off average finger dimensions. The circumference of the metal sheet was equal to the circumference of the support $(4.78 \mathrm{~mm})$.

There were many challenges for simulating the support under the applied point loads. Some of them were: a) introducing point loads in Solidworks ${ }^{\circledR}$, b) size and distribution of mesh, c) type of elements used and d) selecting the linear or nonlinear analysis. In many cases, the von-Mises stress values would continue to increase in magnitude instead of converging at a singular value. Despite this, the displacement values would always be within range of one another.

Introducing the point loads proved to be very complicated due to emergence of singularities which caused divergence in stress values. The best method to work around this was to create small regions of the ring surfaces where the forces were applied. These regions were $0.254 \mathrm{~mm}$ circles to which the forces were then applied.

The other complexity was selecting an appropriate mesh density and the element types. In order to reduce the errors, one should refine the mesh, however, in some cases this leads to divergence. The user should try different meshing techniques or other element types (for example solid elements rather than beam elements) to achieve better results 
The inclusion of an H-adaptive solver achieved converged results (Kurowski, 2016). An H-adaptive mesh is a mesh solver that will automatically refine itself based on the results of the previous study, normally up to five concurrent studies (loops) but additional runs can be added, to achieve a greater degree of convergence ${ }^{11}$. Figure 6 shows an H-adaptive mesh configuration for the solid elements considered.

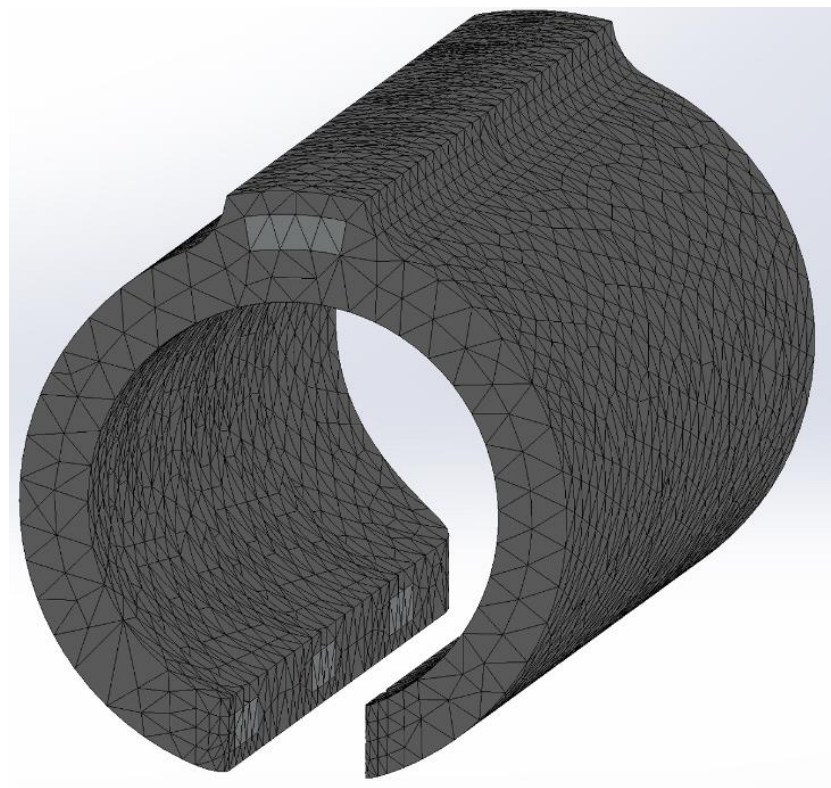

Fig. 6 An H-adaptive mesh with solid elements for the entire model comprised of finger support, metal sheet and three metal rings. For clarity a larger mesh is placed here

In the simulations conducted, target accuracy and accuracy bias were set to maximum in order to achieve converged results. It was found that an initial mesh of $0.762 \mathrm{~mm}$ yielded the best results with the $0.254 \mathrm{~mm}$ surface area loads, mainly because if this initial mesh was refined too much that it would start to mimic a point load at this surface area.

Some nonlinear trials were performed in this study, to predict the large deformations that might occur as a result of an abnormal force. For a thorough nonlinear analysis, one should figure out the Mooney-Rivlin material constants. Additionally, the loads applied should follow the deflection of the beam, because the analysis is transient (Kurowsli, 2016). It was noticed that a linear analysis was adequate to give the predicted results, because of the small amount of displacement that took place. A linear analysis assumes that the stress is proportional to the strain (Kurowski, 2016).

Additionally, a base line was established when comparing the results obtained through this method by considering only the aluminum sheet by itself. Since this is a simple part when compared to the overall assembly, beam elements and point loads were used to gather results and were then verified using the results obtained by MATLAB. A beam element is similar to wire frame geometry where a line is drawn with cross sectional area segments (Kurowski, 2016).

Experimental validation using 3D printing: 3D printing has grown over time as one of the quickest ways to create a product. 3D printing can be more cost effective compared to compression molding, and makes prototyping easier.

The first step was to find the right type of soft polymer to use for the finger support. For this research, TangoPlus-Fullcure-930 (Stratasys ${ }^{\odot}$ ) was first selected because of the reasons mentioned before. The support material was FullCure-705 resin (Stratasys ${ }^{\odot}$ ) and a Polyjet 3D printer was used for production. The sheet and rings were chosen to be made of aluminum.

The first support went through a high pressure water jet cleaning which caused some tears that grew steadily as the resin material was further removed by hand during the washing with low pressure water. The water jet was not used for the second one, and only tab water was used to successfully remove the resin material without any tearing. However, the second one tore by inserting the aluminum rings inside of the cavities. Both supports began tearing at the same spot, which was the edge of the very thin flap where the aluminum ring would enter.

To prevent these issues, instead of using a water jet, a solution of Sodium Hydroxide $(\mathrm{NaOH})$ was used (a liter of the $2 \%$ solution by mixing 20 grams of $\mathrm{NaOH}$ with 980 grams of water). A new part was placed in the solution for 2 hours to resolve the resin material. Also, some dimensions of the support near the rings were modified. Furthermore, it was determined that the part should be tapered to provide a better grip between the support and the finger. This can be accomplished by tapering the entire model or by just having the inside tapered.

Figure 7A shows the fabricated support, validating models four and seven designed in this research. Figure 7B, shows the final model after the-abovementioned modifications.

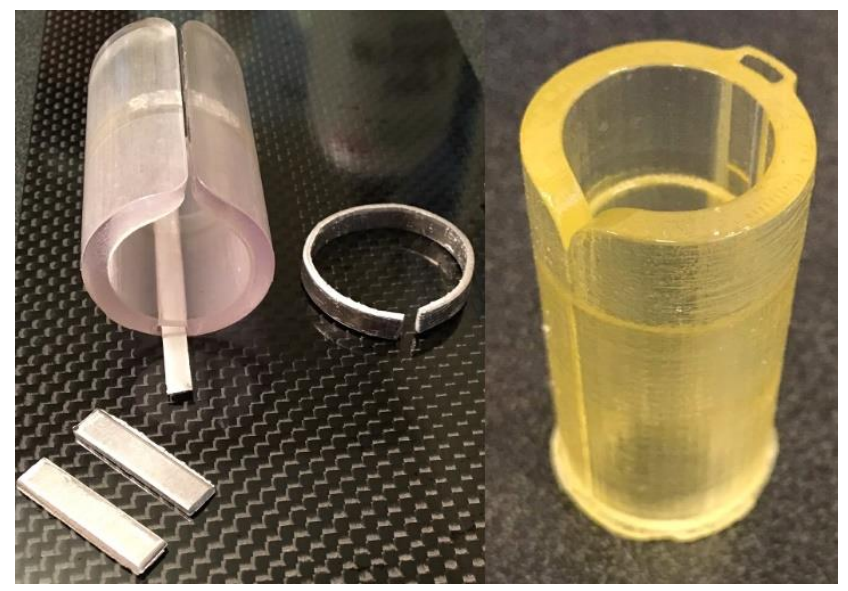

Fig. 7 Finger support model four (7A) and model seven (7B) using TangoPlus and aluminum sheet and rings 
Table 2 The results of simulation for various materials, mesh configurations and element types

\begin{tabular}{cccccc}
\hline \multicolumn{7}{c}{ Finite Element Analysis Setup } & & \\
\hline $\begin{array}{c}\text { Trial No. } \\
\text { \& Vertical Sheet } \\
\text { Mat. }\end{array}$ & Ring Mat. & $\begin{array}{c}\text { Finger Sup. } \\
\text { Mat. }\end{array}$ & $\begin{array}{c}\text { Study and } \\
\text { Solver Type }\end{array}$ & Elem. Type & $\begin{array}{c}\text { Load } \\
\text { Type }\end{array}$ \\
$\begin{array}{c}\text { 1- Al 6061-T6 } \\
\text { (SS)-Flat }\end{array}$ & None & None & Linear Analysis & Beam & Point \\
$\begin{array}{c}\text { 2- Al 6061-T6 } \\
\text { (SS)-Curved }\end{array}$ & None & None & Linear Analysis & Beam & Point \\
$\begin{array}{c}\text { 3- Al 6061-T6 } \\
\text { (SS)- Curved }\end{array}$ & $\begin{array}{c}\text { Al 6061-T6 } \\
\text { (SS) }\end{array}$ & TangoPlus & $\begin{array}{c}\text { Linear Analysis } \\
\text { H-Adaptive }\end{array}$ & Solid & Area \\
$\begin{array}{c}\text { 4- AISI 1020 Steel, } \\
\text { Cold Rolled- } \\
\text { Curved }\end{array}$ & $\begin{array}{c}\text { Al 6061-T6 } \\
\text { (SS) }\end{array}$ & TangoPlus & $\begin{array}{c}\text { Linear Analysis } \\
\text { H-Adaptive }\end{array}$ & Solid & Area \\
$\begin{array}{c}\text { 5- AISI Type H13 } \\
\text { Hot Work Tool } \\
\text { Steel- Curved }\end{array}$ & $\begin{array}{c}\text { Al 6061-T6 } \\
\text { (SS) }\end{array}$ & TangoPlus & $\begin{array}{c}\text { Linear Analysis } \\
\text { H-Adaptive }\end{array}$ & Solid & Area \\
$\begin{array}{c}\text { 6- Carbon Fiber- } \\
\text { Curved }\end{array}$ & Al 6061-T6 & TangoPlus & $\begin{array}{c}\text { Linear Analysis } \\
\text { H-Adaptive }\end{array}$ & Solid & Area \\
\hline
\end{tabular}

Table 3 Maximum values for displacement and stress for each trial compared to the yield (for Carbon fiber the tensile strength is considered). Number of iterations before achieving convergence and the accuracy of the operation are also reported

\begin{tabular}{|c|c|c|c|c|c|}
\hline \multicolumn{6}{|c|}{ Finite Element Analysis Results } \\
\hline $\begin{array}{c}\text { Trial No } \\
\& \\
\text { Vertical Sheet Mat. }\end{array}$ & Max. Displ. & Max. Stress & $\begin{array}{l}\text { Yield Strength } \\
\text { Sheet Mat. }\end{array}$ & $\begin{array}{l}\text { No of Loops to } \\
\text { Conver-gence }\end{array}$ & Accuracy \\
\hline & $(\mathrm{mm})$ & (MPa) & (MPa) & & \\
\hline 1- Al & 0.554 & 42.06 & 275 & 1 & \\
\hline 2- Al & 0.586 & 52.1 & 275 & 1 & \\
\hline 3- Al & 0.554 & 45.8 & 275 & 13 & 99.995 \\
\hline 4-CR Steel & 0.204 & 43.7 & 350 & 13 & 100 \\
\hline $\begin{array}{c}\text { 5- Hot Work Tool } \\
\text { Steel }\end{array}$ & 0.199 & 42.8 & 1650 & 17 & 100 \\
\hline 6- Carbon Fiber & 0.187 & 47.8 & 2800 & 9 & 99.995 \\
\hline
\end{tabular}

Table 4 Estimating the maximum forces that each support can tolerate by changing the sheet metal insert

\begin{tabular}{|c|c|c|c|c|c|c|c|c|}
\hline \multicolumn{9}{|c|}{ MATLAB Calculated Factor of Safety } \\
\hline \multirow{2}{*}{$\begin{array}{l}\frac{3}{2} \\
\frac{2}{2} \\
\frac{2}{2}\end{array}$} & \multirow{2}{*}{ 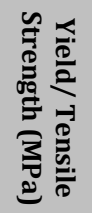 } & \multirow{2}{*}{ 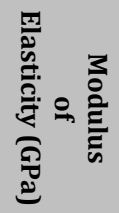 } & \multirow{2}{*}{ 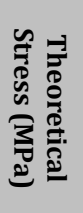 } & \multirow{2}{*}{ 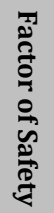 } & \multicolumn{3}{|c|}{ 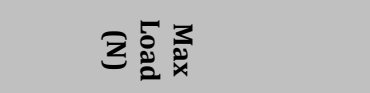 } & \multirow{2}{*}{ 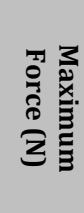 } \\
\hline & & & & & 1 & 2 & 3 & \\
\hline Al & 275 & 69 & 42.06 & 6 & 7.9 & 5.27 & 2.64 & 15.82 \\
\hline CR Steel & 350 & 205 & 42.06 & 8 & 10.1 & 6.71 & 3.35 & 20.13 \\
\hline Tool Steel & 1650 & 210 & 42.06 & 39 & 47.5 & 31.62 & 15.81 & 94.9 \\
\hline $\begin{array}{c}\text { Carbon } \\
\text { Fiber }\end{array}$ & 2800 & 228 & 42.06 & 66 & 80.6 & 53.66 & 26.83 & 161.1 \\
\hline
\end{tabular}

\section{Results}

This research introduced a new finger support, which allows patients to have a comfortable and flexible support to wear on their finger with the goal to eventually decrease the pain and increase the ability to complete everyday tasks. Composite models made of a polymer and metal (aluminum, steel, or carbon fiber strips) were designed and two of them were manufactured through 3D printing.
The rubber-like material which was selected was TangoPlus-Fullcure-930, a soft polymer that decreases the amount of irritation on the skin compared to other available harder or uncomfortable products. 3D printing was chosen for production.

Seven different models were designed: fibers, slotted, tapered, flexible, angled, versatile, and optimal The fibers model is more difficult to manufacture because of the limitation of a normal Polyjet printer. The slotted and tapered supports do not allow all finger sizes; although they provide the best grip for the 
finger and they are very easy and fast to manufacture. Flexible and angled supports accommodate adjustments and functional finger position respectively; however they are not as strong as the last two models. The versatile model is useful for functional position and accommodates more angles of bend compared to the angled one. It allows many people to be able to adjust the support to their type of finger deformity and control the bent finger. The optimal model is the strongest and tolerates higher finger forces and can be modified to become tapered to provide more grip and to be used in functional finger positions.

Figure 8 shows the results of simulation for the optimal model considering aluminum for the sheet and rings. The von-Mises stress values and the deformations are shown for the forces mentioned before. It is necessary to mention that the values obtained for all metals are far from the yield strength of each metal tested (see Table 3).

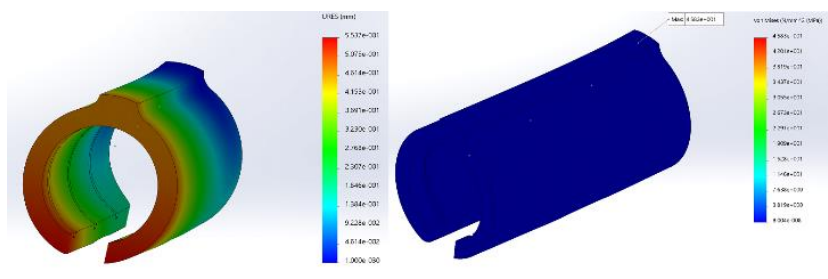

Fig. 8 Displacement throughout the composite support (Left or 8A) and von-Mises stress values for the finger loads applied (Right or 8B)

Table 2 shows the setup of each simulation, while Table 3 records the overall results of the FEA. It should be noted that trial number 1 and 2 were simple cantilever beam analyses without the rings or TangoPlus finger support. Additionally, the accuracy for trial numbers 3 through 6 were the target accuracy provided by SolidWorks ${ }^{\odot}$. For carbon fiber, the tensile strength is considered for comparison, because in most cases this brittle material has no yield strength.

A MATLAB program was written to verify trial 1, which was then used to give an idea of the validity of the other studies performed. Equations 3 and 4 were used to test a flat vertical sheet using the forces, and distances mentioned in the modeling portion of this paper. The beam was $4.78 \mathrm{~mm}$ by $1.60 \mathrm{~mm}$ by $50.80 \mathrm{~mm}$, and aluminum 6061-T6 was considered with a modulus of elasticity of $69000 \mathrm{MPa}$.

These calculations yielded a maximum stress of 42.057 MPa at the fixed end of the sheet, while a maximum displacement of $0.553 \mathrm{~mm}$ was found. These values were then compared to trial 1 and were virtually the same as the simulation results; a maximum stress of $42.1 \mathrm{MPa}$ and maximum displacement of $0.554 \mathrm{~mm}$ respectively.

The rest of the simulations used an $\mathrm{H}$-adaptive solver, with an initial mesh of $0.762 \mathrm{~mm}$, and calculated the maximum von-Mises stress and the maximum displacement. It was determined that aluminum 6061-
T6 vertical beam experienced the greatest von-Mises stress of 45.8MPa, while AISI Type H13 hot work tool steel experienced the least at $42.8 \mathrm{MPa}$. The maximum displacement was again found in the aluminum vertical sheet $(0.554 \mathrm{~mm})$ and the lowest one was found in the one with carbon fiber $(0.187 \mathrm{~mm})$.

The best method to determine if the above stress values are acceptable, is to compare with the yield strength of the material if a ductile material is used such as aluminum. This is also known as the von-Mises Hencky theory (Kurowski, 2016). According to the maximum normal stress failure criterion, the vonMises stress values obtained should be evaluated against the tensile strength, if the material is somewhat brittle or has no yield point (Kurowski, 2016). All of the results showed that the maximum stress was well below the yield strength of the material which proved that the finger support would not fail under the loads applied.

Lastly, the MATLAB code was used to approximate the maximum load that different support materials can handle. This was done by considering the yield, or tensile, strength of the material against the theoretical stress values to find the factor of safety. This factor of safety was then multiplied by the initial loads (1.21, 0.806 , and 0.403 -Newtons) to find the maximum loads capable. The results of this MATLAB analysis are recorded in Table 4.

\section{Discussion}

Design and fabrication of a finger support, which is comfortable and resizable, yet accommodates various finger positions, has not been addressed by the research community. By introducing new finger supports, this work will be able to further advance the current limited existing products for finger deformities. The goal of this work has been to design the devices to support the painful joints and make sure that they are positioned correctly. The support should straighten the finger and/or correct its abnormal curvatures. These can be designed for the resting position to ease pain and inflammation during a painful flare-up or a period of unusual discomfort.

Some others should modify the severely bent fingers, into a functional or working position of fingers (when fingers and thumb are in flexion) to make the job less painful. These can be worn when a person carries out daily tasks.

In this work, seven support models were designed, and advantages and disadvantages of them were reviewed. The last two models (versatile and optimal), are stronger, and resizable. Each of these two supports was made of a soft polymer with inserted metal sheets and rings. The idea of combining the materials and making a composite model was to obtain many advantages at the same time. The support should be soft and comfortable, yet very strong to tolerate the unpredictable loads. Various metals were considered to compare the maximum stress and deformation that 
they could handle. The maximum force that each support can tolerate is reported. The optimal model tolerates up to a total of about 161-Newtons if a carbon fiber insert is used, which has not been achieved elsewhere. It can be modified to be used in functional finger position.

The extensive modeling portion of this research proved to be the most difficult as convergence was a challenge to achieve. However, after identifying the causes of singularities, more efficient methods were selected and therefore better results were obtained. The modeling, overall, had a great learning curve as many criteria and methods were reviewed and evaluated as discussed in the modeling section of this paper. The fact the simulation was done using a linear analysis proves that the support was designed very well because the deformations were not plastic.

Another point of discussion is related to meshing which was a source of problem when combined with both point loads and the small-area loads. It would be interesting to study these relationships furthermore, to optimize the analysis for future work.

A fatigue analysis of the finger support would also be beneficial for research purposes in determining how long the finger support can withstand the fluctuating loads during a typical day.

The design was evaluated through the experiment and fabricated with many parts. It is suggested to either modify the dimensions, so the support can withstand the steps involved in fabrication or change the polymer to either TangoBlackPlus or Agilus30-FLX. Agilus30-FLX has a higher tensile tear resistance $\left(5-7 \mathrm{~kg}-\mathrm{cm}^{-1}\right)$ compared to TangoPlus-930 $\left(2-4 \mathrm{~kg}-\mathrm{cm}^{-1}\right.$, which is low compared to other polymers from its family). The Agilus30 family provides superior tearresistance, elongation at break and rubber-like texture. Furthermore, if adjustment is not an issue, a model similar to model three with the capabilities of model six would be ideal. This model would have a better grip on the finger and is also versatile and could be bent to accommodate any finger position.

Another design that can be created is the composite model with metal fibers, which will make the finger support stronger with or without inserting strips of metal. When creating a composite, it would be required to use a Connex 3D printer that is capable of printing with multi-material. This option can be explored by other researchers in the future.

Finally, the authors believe that the new finger supports which were designed and fabricated, can be used by patients and overall this approach will open the door for other researchers to select other manufacturing processes and try out more complicated models.

\section{References}

Y. Z. Arslan, Y. Hacioglu, N. Yagiz (2008), Prosthetic hand finger control using fuzzy sliding modes, Journal of Intelligent and Robotic System, Vol.5, No.1, pp.121-138.
J. W. Brandsma, J. M. Watson, (1982), Clawfinger correction: results of extensor-flexor many tailed operation, The Hand, Vol.14, No.3, pp.307-12.

J. Colbourn, N. Heath, S. Manary, D. Pacifico, (2008), Effectiveness of splinting for the treatment of trigger finger, Journal of Hand Therapy, Vol.21, No.24, pp.336-343.

A. Deveci, S. Yilmaz, A. Firat, A. Ucaner, (2015), An overlooked deformity in patients with hallux valgus tailor's bunion, Journal of American Podiatric Medical Association, Vol.105, No.3, pp.233-237.

P. Egger, C. Cooper, D. J. Hart, D. V. Doyle, D. Coggon, T. D. Spector, (1995), Patterns of joint involvement in osteoarthritis of the hands: the chingford study, $J$ Rheumatol, Vol.22, No.8, pp.1509-1513.

G. Foucher, C. Lequeux, J. Medina, D. Nagel, (2001), A congenital hand deformity: dupuytren's disease, Journal of Hand Surgery, Vol.26, No.3, pp.515-517.

F. J. Van Der Giesen, W. J. Van Lankveld, C. Kremers-Selten, A.J. Peeters, E.B. Stern, S. Le Cessie, R. G. H. H. Nelissen, T. P. M. Vliet Vlieland, (2009), Effectiveness of two finger splints for swan neck deformity in patients with rheumatoid arthritis: a randomized, crossover trial, Arthritis \& Rheumatism, Vol.61, No.8, pp.1025-1031.

M. P. Groover, (2010), Fundamentals of Modern Manufacturing, $4^{\text {th }}$ ed. Hoboken: John Wiley \& Sons, Inc, pp. 153-186.

S. Han, X. Jin, F. Costa, A. Melnikov, (2014), The numerical and experimental study on the compression and transfer molding of rubber materials, AIP Conference Proceedings, Vol.1593, No.1, pp.508-511.

A. Hofmann, W. Goebl, (2016), Finger forces in clarinet playing, Frontiers in Psychology, Vol.7, pp. 1140.

P. M. Kurowski, (2016), Engineering Analysis with SolidWorks Simulation 2016, Mission: SDC Publications, pp. 5-30 \& 211-227 \& 238-281.

C, Padiachee, F. Shahid, N. Jamayet, M. Alam, (2016), Management of mallet finger injury by alam prosthetic device (APD, International Medical Journal, Vol.23, No.6, pp.700-701.

N. S. Palchik, D. M. Mitchell, N. L. Gilbert, A. J. Schulz, R. F. Dedrick, T. D. Palella, (1990), Nonsurgical management of the boutonniere deformity, Arthritis \& Rheumatology, Vol.3, No.4, pp.227-232.

S. N. P. da Silva, S. Nicolau, R. Mattar, R. B. Neto, C.A.M. Pereira, (2005), Measurement of the Flexing Force of the Fingers by a Dynamic Splint with a Dynamometer, Clinics, Vol.60, No.5, pp. 381-388.

K. Valdes, N. Naughton, L. Algar, (2015), Conservative treatment of mallet finger: a systematic review, The Journal of Hand Therapy, Vol.28, No.3, pp.237-246.

A. V. Vasiliadis, I. Itsiopoulos, (2017), Trigger finger: An atraumatic medical phenomenon, Journal Hand Surgery Asian Pac, Vol. 22, No.2, pp.188-193.

L. Wang, T. Meydan, P. Williams, (2017), Design and evaluation of a 3D printed optical sensor for monitoring finger flexion, IEEE Sensors Journal, Vol.17, No.6, pp.1937-1944.

K. Williams, A. L. Terrono, (2011), Treatment of boutonniere finger deformity in rheumatoid arthritis, Journal of hand surgery, Vol.36, No.8, pp.1388-1393. 